\title{
Pointing System for the Large Size Telescopes Prototype of the Cherenkov Telescope Array
}

\author{
Darko Zarićc, ${ }^{* a}$, Stefan Cikota ${ }^{b}$, Armand Fiasson $^{c}$, Nikola Godinović ${ }^{a}$, Koji Noda $^{d}$, \\ Robert Wagner $^{e}$, Martin Will $^{f}$, Quentin Piel $^{c}$ for the CTA LST project ${ }^{\dagger}$ \\ ${ }^{a}$ Faculty of Electrical Engineering, Mechanical Engineering and Naval Architecture \\ University of Split, 21000 Split, Croatia \\ ${ }^{b}$ Faculty of Electrical Engineering and Computing, University of Zagreb, 10000 Zagreb, Croatia \\ ${ }^{c}$ Laboratoire d'Annecy de Physique des Particules, \\ Univ. Grenoble Alpes, Univ. Savoie Mont Blanc, CNRS, LAPP, 74000 Annecy, France \\ ${ }^{d}$ Institute for Cosmic Ray Research, the University of Tokyo, 277-8582 Chiba, Japan \\ ${ }^{e}$ Oskar Klein Centre, Department of Physics, Stockholm University, Albanova University Center, \\ SE-10691 Stockholm, Sweden \\ ${ }^{f}$ Max-Planck Institute for Physics, D-80805 Munich, Germany \\ E-mail: Darko.Zaric@fesb.hr
}

The pointing system of the prototype of the Large Size Telescope (LST-1) for the Cherenkov Telescope Array observatory, should ensure mapping of the gamma-ray image of a point-like source in the Cherenkov camera to the sky coordinates with a precision better than 14 arcseconds. Detailed studies of the telescope deformations are performed in order to disentangle different deformations and quantify their contributions to the miss-pointing, to learn how to correct for them, and finally how to design the system for offline and online pointing corrections. The LST-1 pointing precision system consist of several devices mounted at the center of the dish: Starguider Camera (SG), Camera Displacement Monitor (CDM), two inclinometers, four distance meters, and an Optical Axis Reference Laser (OARL), working together with the LEDs mounted in a circle around the Cherenkov camera. The online pointing corrections are based on a bending model as currently done by existing IACTs. The offline corrections will be performed combining measurements done by the SG and CDM cameras. SG will provide the position of the Cherenkov camera center with respect to the sky coordinates with a precision of 5 arcseconds, while CDM will provide the deviation of the telescope optical axis defined by the OARL spots with respect to the Cherenkov camera center with a precision better than 5 arcseconds. Laboratory measurements on dedicated test benches showed that the required pointing precision can be achieved for SG, CDM and inclinometer.

36th International Cosmic Ray Conference -ICRC2019-

July 24th - August 1st, 2019

Madison, WI, U.S.A.

\footnotetext{
${ }^{*}$ Speaker.

${ }^{\dagger}$ for consortium list see PoS(ICRC2019)1177
} 


\section{Introduction}

The prototype of the Large Size Telescope (LST-1) of the Cherenkov Telescope Array (CTA) has been installed in 2018 at the north CTA site located at the Roque de los Muchachos Observatory on La Palma in the Canary Island and its commissioning should be finished in 2019 [1].

The precision with which a Cherenkov camera coordinate in the focal plane of the CTA telescopes can be mapped to a celestial coordinate is called the telescope pointing precision. Knowing the Cherenkov camera position very precisely during favourable observing conditions is needed to achieve the performance requirements of CTA[2]. Under favourable observing conditions the rootmean-square (RMS) systematic error on the localisation of a point-like source of gamma rays below $100 \mathrm{GeV}$ per axis must be $<10^{\prime \prime}$ per axis. To achieve this the rms space-angle post-calibration pointing precision for the LST telescopes must be $<14^{\prime \prime}$.

The LST design is conceived to provide a large photon collection area sensitive enough to have a low energy threshold of $20 \mathrm{GeV}$, while the structure should be light as possible to allow a fast reaction to transient events. To achieve these two objectives it is a matter of trade-off, as large collection area needs a big (i.e. heavy) structure, but fast re-positioning requires a light structure which on the other hand leads to deformable structure affecting the telescope pointing precision. Due to experiences with the currently working IACTs we already know that we cannot build a perfectly stiff telescope with a reasonable weight. The obtained data must be corrected for all the deformations in the telescope component. There are several types of deformations that can occur on the telescope such as deformations due to gravity, temperature change, stable wind or by a wind gust. Currently in the MAGIC telescopes only gravity [3], [4] has been considered in the correction methods and the rest of the deformations are corrected for an online correction called "drive bending model" and with an offline precise correction by a Starguider system.

\section{Deformations of the LST}

The bending model alone is not enough to correct for the deformations by a temperature change, static and dynamic winds, as these three effects cannot be easily reproduced in advance. A gust or the dynamic component of a continuous wind can change in a time scale faster than a few seconds. Since Starguider system will be able to provide corrections with a rate less than a $\mathrm{Hz}$, another system is needed to account for this deformations. In order to separate the camera displacement from the deflection in the dish structure, Optical Axis Reference Laser (OARL) spots will be used as a reference, and then the camera positions will be measured with the help of Reference LEDs. To correct for the effect by a gust which will have oscillations of $\sim 2 \mathrm{~Hz}$, dedicated Camera Displacement Monitor (CDM) with a rate of $10 \mathrm{~Hz}$ will be installed. This means that OARL will serve as a reference to the dish structure, inclinometers will relate it to the drive system and CDM will relate it to the camera [5].

It is important to identify all structural deformations contributing to telescope mispointing and to disentangle them as much as possible using various monitoring techniques. Apart from global misalignment of the telescope's azimuth and elevation axes, pointing deviations are expected due to structural deformations of the telescope structure, such as: 
(1) a rotation or twist of the telescope structure in azimuth direction (not taken account for by the drive assembly)

(2) a twist of the elevation towers

(3) a twist between the elevation structure (dish and arc) and the elevation motor sitting in the lower telescope structure

(4) a deformation of the mirror dish

For item (1) this deformation is expected to make only a slow common deflection to the dish and the arch, thus it can be corrected for with the Starguider. For (3) inclinometers are installed at the dish center and they will be firmly attached to the OARL and in that way they will directly measure the inclination angle at the dish. The deformations (4) by the static causes (the gravity, temperature and static wind) can be corrected for by Active Mirror Control (AMC). Furthermore, deformation of the arc structure may affect the relative orientation of the photomultiplier-tube (PMT) camera w.r.t.the telescope's optical axis, shown in figure 1, such as:

(i) a vertical or horizontal shift of the focal plane

(ii) a rotation of the focal plane around the optical axis

(iii) tilts of the focal plane w.r.t. the optical axis

(iv) a shift along the optical axis.

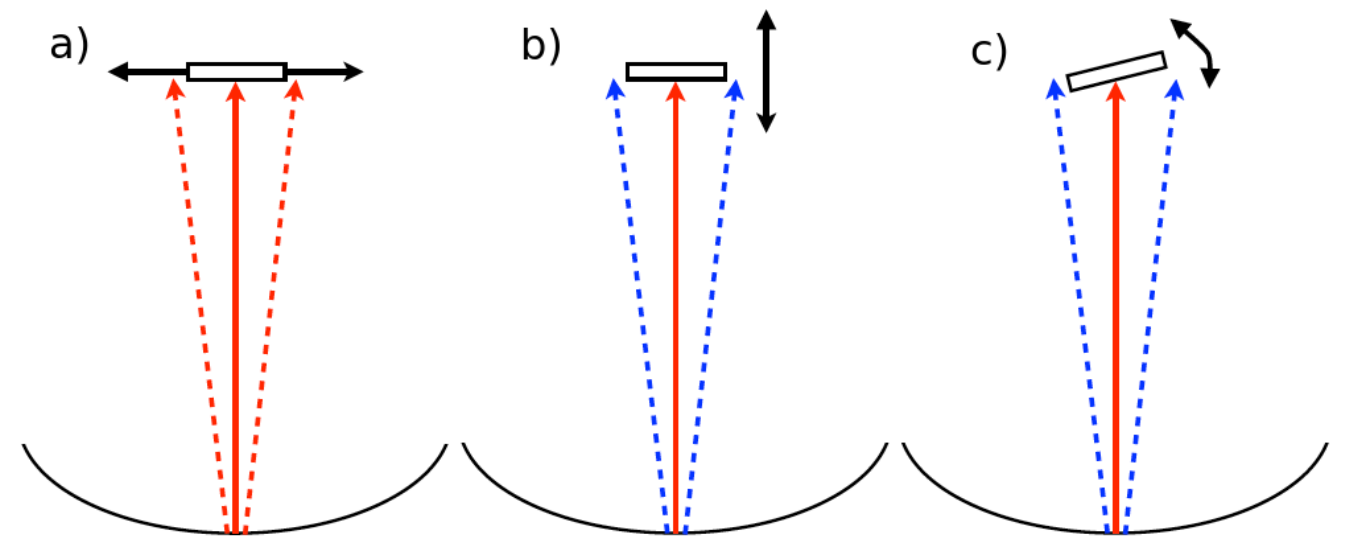

Figure 1: Deformation between the dish and the camera. a) Displacement on the focal surface for $x$ - and $y$-axes (and rotation around z-axis. b) Change of the focal length along z-axis, and c) Tilting of the focal surface, rotations around $\mathrm{x}$ - and $\mathrm{y}$-axes

For item (iii) and (iv) four distance-meters are be installed at the dish centre, to measure the distance to the camera plane, and also its tilting angle.

Finally we will correct for all the effects by Starguider, which uses the sky field as the reference, to achieve the final precision less than 14 arc-seconds. 
Elastic deformations can be well parametrised by a bending model prepared in advance (using special calibration observations where the telescope is pointed at several bright stars homogeneously distributed in the azimuth-elevation plane). Such bending model, however, cannot correct for inelastic deformations of the telescope structure (e.g. due to temperature changes, constant or gusty wind loads). To take these effects into account in the pointing calibration, deformations have to be recorded as much as possible during science data taking. For the LST, installation of the dedicated monitoring devices is foreseen.

\section{Hardware components}

\subsection{Reference LEDs}

Reference LEDS are installed around the PMT camera and are used as a reference, even during data acquisition (DAQ). There are 12 LEDs mounted in a circle around the PMTs, and 3 more are located in each corner of the PMT camera hood as seen in figure 2. The LEDs are ROHM SLI343V8R3F with a dominant wavelength of $630 \mathrm{~nm}$ and on each LED there is a cap with a collimated hole with a size of $1 \mathrm{~mm}$.

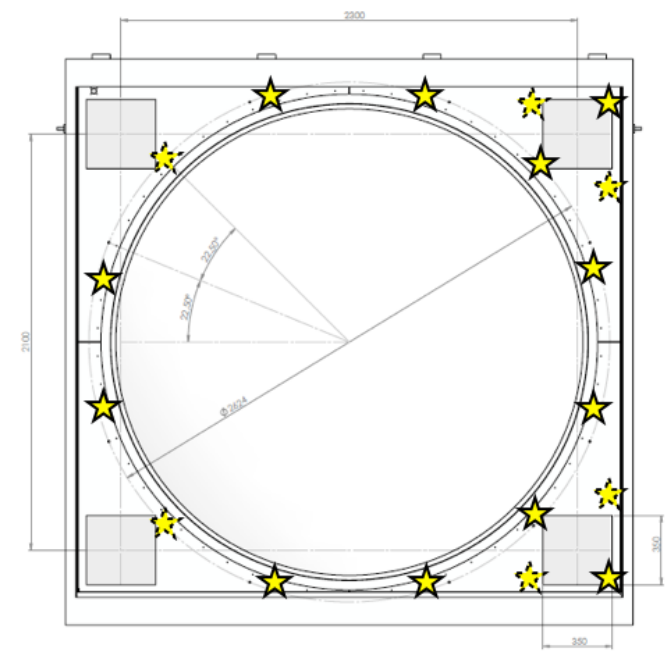

Figure 2: Position of the LEDs on the lid of the PMT camera.

\subsection{Starguider}

This camera corrects for the telescope pointing with a high precision, using known stars in the catalogues. It sees a star field, and also a part of Reference LEDs. Since the Starguider camera will be located in the dish centre, its line of sight will be inclined by a few degrees w.r.t. the telescope's optical axis. The Starguider can know only the relative position of the camera in the sky field, which does not necessarily correspond to the pointing direction of the telescope dish. In fact, a Finite Element Analysis for the LST structure [6] shows that, the dish and the arch deform differently, strongly dependent on the direction of the wind. For that reason CDM is used.

While the LST drive system tracks the source, every second the SG camera takes the images of stars 
in its field of view close to the tracking source. Images of stars taken by SG camera are analyzed to identify the star and their relative position using the star catalogue to provide mapping of the SG pixels to sky coordinates and the number of correlated stars. Since the SG camera also measures the center of the PMT camera (part of the LEDs mounted on circle around the PMT camera are also in the SG camera field of view) and the mapping between the SG camera pixels and the sky coordinates is known, the sky coordinates of the PMT camera are also known. The Starguider correction will be applied offline.

For the SG a Peltier cooled low-noise camera from Allied vision was chosen. Bigeye G-283 Cool has resolution of $1928(\mathrm{H}) \times 1452(\mathrm{~V})$ with pixel size 4.54 microns $x 4.54$ microns. The Kowa LM50HC lens of $50 \mathrm{~mm}$ focal lens gives the field of view (FoV) of $9.938 \mathrm{deg}$ (horizontal) $\mathrm{x} 7.485$ deg (vertical), which results with a resolution of $18.56 \mathrm{arcsec} / \mathrm{pix}$. At $28 \mathrm{~m}$ the FoV measures 4.90 $\mathrm{m}$ x $3.66 \mathrm{~m}$. The software to control SG camera is used over OPC Unified Architecture (OPCUA) server enabling it to take images, control exposure and send images over the Ethernet link to the defined IP address.

\subsubsection{Analysis procedure of the Starguider}

The main purpose of the LST Starguider is to find the center of field of the LST. The first step towards that is to search the center of field of the star guider camera. The main tools used for image solving and searching the center of field are the WCSTools [7], [8]. WCSTools is a package of programs and a library of utility subroutines for setting and using the world coordinate systems (WCS) in the headers of the most common astronomical image format, the FITS format, to relate image pixels to sky coordinates. This software is all written in very portable C. The WCSTools commands are executed in terminal. Because the LST star guider software consists of a set of different WCSTools procedures, a script in Python was written that executes the WCSTools commands in terminal. Determining the center of field of an individual image by using the WCSTools is done with the utility "immatch". The "immatch" utility is used for matching stars in a FITS (or IRAF image) with a world coordinate system (WCS) in its header with stars in a reference catalog. The WCS is the relationship between sky coordinates and image pixels and can be described in a standard way in the header of an image. The header of our star guider FITS images was set to be compatible with the standard WCS format while taking (saving) the images. The utility immatch supports different star catalogues: The HST Guide Star Catalog, the USNO UJ Catalog, the USNO A Catalogs, or user-supplied catalogs may be used. For software tests with images that were collected at the public observatory Mosor (in the mountains near Split, Croatia), the HST Guide Star Catalog was used. The immatch utility takes between 5 and 8 seconds to reduce the individual images, match the extracted stars with the star catalogue and find the center of field. Since the main requirement of the LST star guider software is to determine the center of field of individual images within 1 second, the immach utility by itself is too slow, and a different approach was established. Since the LST telescope is not changing its field of view during the data acquisition of a particular target, performing the immatch utility can be avoided for each individual image, and can instead be performed only after every reposition of the LST telescope, while the other star guider images during LST data acquisition will be processed by using SExtractor and Python's SciPy kd-tree tool for quick nearest-neighbor lookup. A sketch of the procedure used in the LST star guider script is given in figure 3. 


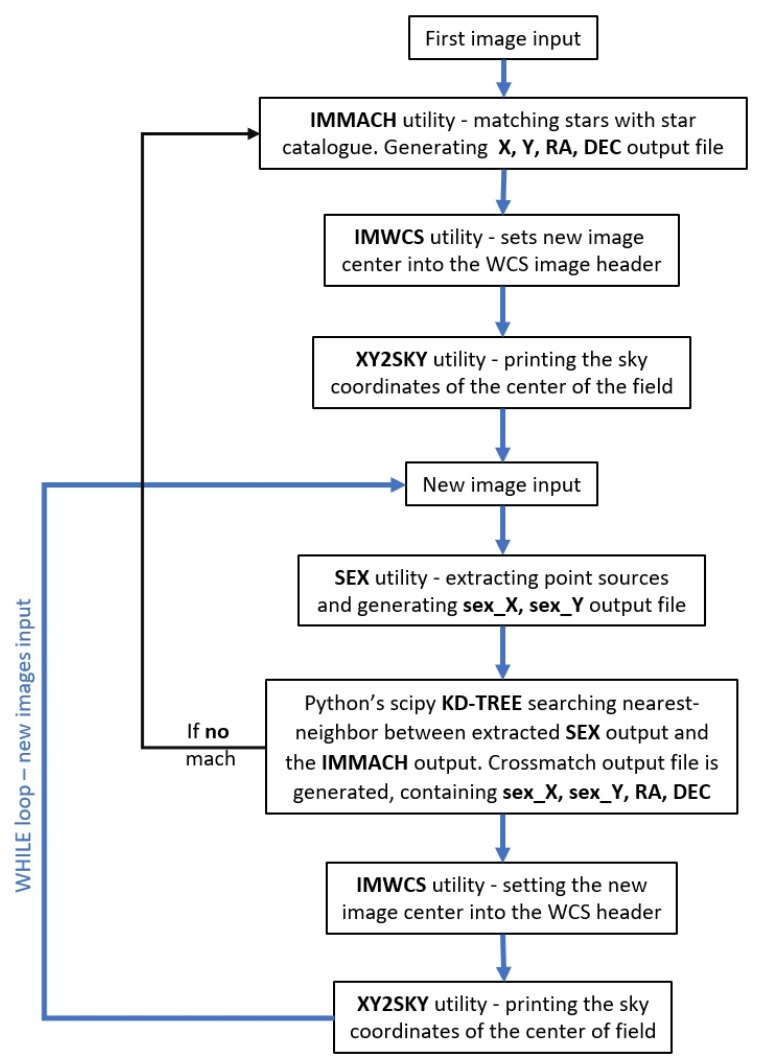

Figure 3: Sketch of the LST starguider software procedure

\subsection{Optical Axis Reference Laser}

OARL defines the optical axis of the LST during science observations. Two OARLs are installed in order to provide 2 dimensional information. They are installed at the dish centre, pointing towards screen targets at the edges of the PMT camera. When observed by the CDM, the two reference spots can be compared to the reference LEDs, providing a correction for the PMT camera displacement. A stiff unit is built, in which the inclinometer is sandwiched by the two OARLs, to represent the dish structure. The unit will be installed at the center of the dish with a dummy facet in order to avoid any additional systematic deformation at the central hub. 2 infrared laser chosen as the OARL are made by ProPhotonix. Their wavelength of the emitting light is $780 \mathrm{~nm}$, the power output is $3 \mathrm{~mW}$ and they are focusable.

\subsection{Inclinometers}

The inclinometers will be used to measure dish deformations during data taking. Finite element analysis of the LST structure has confirmed that the inclination at the dish center is closely representing that of the whole dish structure. Therefore, it is planned to measure directly the inclination angle at the dish center, by inclinometers firmly attached to OARL. The inclinometer will be combined with OARL in the hardware level. There are two inclinometers ("Tuff Tilt Digital" by Jewell Instruments), one for the elevation angle (parallel to the optical axis) and one parallel to 
the elevation axis. The former one has a wide range of $\pm 50^{\circ}$ with 14 arsec of precision, while the latter one has more narrow range $\pm 3^{\circ}$, but with a higher precision of 1 arsec.

\subsection{Camera Displacement Monitor}

Camera Displacement Monitor (CDM) will be located in the central hole of the dish and monitor during science observations the horizontal and vertical displacement of the PMT camera, as well as the rotation of the focal plane w.r.t. optical axis defined by the OARL. It will see OARL and Reference LEDs in its FoV. It will use a frame rate of $10 \mathrm{~Hz}$ and it will separate the camera displacement from the dish deformation. The CDM correction will be used offline.

The CDM system is built of: camera, lens, processing unit and a sensor for measuring temperature, pressure and humidity. Processing unit is Intel NUC Mini PC NUC5i7RYH running CentOS. Camera is produced by Imaging Development Systems (IDS), and the model is UI-3590CP, which is a CMOS camera connected with USB 3.0 to the processing unit. It can achieve frame rates up to $21.0 \mathrm{fps}$ and its maximal resolution is $4912 \times 3684$ which amount to $18.10 \mathrm{MPix}$. The IR filter has been removed from the camera so it can be sensitivity to the OARL spots. It is equipped with Edmund Optics 35mm lens. Having focal length of $35 \mathrm{~mm}$ provides the horizontal FoV of $7 \mathrm{deg}$ and vertical of 9 deg and a sampling of $7 \mathrm{arcsec} /$ pixel.

\subsubsection{Bending model}

The same camera will be used for generating the bending models. During the pointing calibration observations it will observe the Reference LEDs on the PMT camera and the image of the bright star projected on a reflective screen in front of the PMT camera. The only difference is that in this mode the camera will take images with longer exposition times. It will be controlled using the the ACS (ALMA Common Software) framework and the OPCUA protocol provided by ACTL (Array Control and Data Acquisition System).

A star will be tracked with the available initial conditions. Its direction $(\mathrm{Zd}, \mathrm{Az})$ is calculated from (Ra, Dec), and ( $\mathrm{Zd}, \mathrm{Az})$ is converted to the motor steps with the encoders. The star spot will be visible on the central screen with a shift with respect to the optical axis (defined by the OARL spots). The shift values taken with all the tracked stars in a large range of $(\mathrm{Zd}, \mathrm{Az})$ are stored with the encoder and inclinometer measurements. Then we can generate a bending model, a relation from the encoders and inclinometers to the optical axis. The correction will be used online during DAQ.

Parameters to be used in the bending model would be as follows 1) trivial offsets between the axes and encoders, 2) azimuth axis misalignment, 3) non-perpendicularity of the azimuth and elevation axes, 4) non-perpendicularity of the elevation and optical axis, 5) small non-centricity of the axes, 6) a zenith-angle dependent deflection of the optical axis.

\subsection{Distancemeter}

Distancemeters (DMs) measure the distance and tilting of the focal plane w.r.t. the dish centre. In general measurements for a $28 \mathrm{~m}$ distance utilize a time-of-flight sensor with a laser. There are 4 DMs (model DL35 made by SICK) that use infrared lasers (laser class 1) in order not to affect the DAQ by PMTs. They each point in the corner of the PMT camera lid and they each have $\pm 15 \mathrm{~mm}$ of accuracy. 


\section{Outlook}

Drawback of two camera solutions, SG camera pointing to the sky and CDM pointing to the PMT camera, to achieve a LST pointing accuracy of 14 arc seconds, is the necessity to perform the careful inter-calibration between the cameras. Namely the displacement of the telescope optical axis in respect to the PMT camera centre has to be translated in the sky coordinates of the PMT camera centre measured by SG camera. The transformation matrix between SG camera coordinates and CDM coordinates will be determined from the measured coordinates of the same six LEDs seen by both cameras following the procedure [9]. The software tool to inter-calibrate these two cameras will be tested in the laboratory first by taking image by both cameras of the same 6 LED seen by both cameras without any tilt between them and then with the tilt of 3 degree as it is expected upon mounting on the telescope. Once the housing with pointing system on the LST dish and proper tilt between two cameras is found out, the calibration procedure tested by the laboratory measurements will be done by taking several thousand images and running on the already developed and tested software for the camera inter-calibration. It is expected that the pointing system will be installed at the LST-1 by the end of August 2019.

\section{Acknowledgments}

This work was conducted in the context of the CTA LST Project. We gratefully acknowledge financial support from the agencies and organizations listed here:

http: //www.cta-observatory.org/consortium_acknowledgments and the support by the Croatian Science Foundation (HRZZ) Project IP-2016-06-9782.

\section{References}

[1] J. Cortina for the CTA consortium, Status of the Large Size Telescope of the Cherenkov Telescope Array, in proceedings of 36th International Cosmic Ray Conference, PoS(ICRC2019) (2019).

[2] www.cta-observatory.org/project/technology

[3] T. Bretz, D. Dorner, R. Wagner, and MAGIC collaboration, The tracking system of the MAGIC telescope, in proceedings of 28th International Cosmic Ray Conference (Vol. 5, p. 2943). (2003).

[4] P.T. Wallace, TPOINT-Telescope Pointing Analysis System, Starlink User Note 100, 1994.

[5] K. Noda, Alignment and pointing corrections and procedures, CTA LST/140721 (internal publication)

[6] K. Noda, LST pointing and alignment performance with the finite element analysis, CTA LST/20140729 (internal publication)

[7] D. J. Mink, WCSTools: Image World Coordinate System Utilities, In Astronomical Data Analysis Software and Systems VI (Vol. 125, p. 249). (1997).

[8] D. J. Mink, Browsing Images in World Coordinate Space with SAOimage, In Astronomical Data Analysis Software and Systems V (Vol. 101, p. 96). (1996).

[9] J. Cashbaugh, C. Kitts: Automatic Calculation of a Transformation Matrix Between Two Frames, IEEE Access 6 9614-9622. (2018) [10.1109/ACCESS . 2018.2799173] 